\title{
Underdiagnosis of respiratory diseases during an economic downturn and the need for spirometry as a screening test
}

Subdiagnóstico de doenças respiratórias durante uma desaceleração econômica e necessidade do uso da espirometria como teste de triagem

\author{
Filio Kotrogianni, Foteini Malli, Konstantinos 1 Gourgoulianis
}

\section{To the Editor:}

In a very interesting paper published recently in the Brazilian Journal of Pulmonology, Queiroz et al.(1) reported a COPD underdiagnosis rate of $71.4 \%$ in a primary health care setting. In addition, the authors observed that only 5.6\% of the subjects previously diagnosed with COPD had undergone pulmonary function tests (PFTs). We would like to recount our experience concerning the underutilization of diagnostic tools, such as spirometry, and the consequent underestimation of respiratory diseases. The underdiagnosis of major health problems, such as asthma and COPD, has to be noted, especially in a period during which access to health care is no longer a universal, inalienable right, because of the recent economic downturn.

Greece is currently dealing with a major financial crisis, which has had disheartening effects on every aspect of life, including not only access to health care but also the quality of the health care services available. In particular, the reduction in family income and the inability of individuals to afford health insurance coverage, has limited the use of high-quality health care services. The total government expenditure on (budget for) health care has been also affected by the economic crisis. The World Health Organization reported that, due to the economic downturn, patients in Greece are either seeking medical assistance at public health care facilities or deferring health care completely. ${ }^{(2)}$ As a result, an increasing number of patients remain undiagnosed even if they are symptomatic or have specific risk factors for a particular disease, such as COPD.

Our group, in cooperation with the Hellenic Thoracic Society, evaluated 267 individuals (183 males; mean age: $53.5 \pm 14.3$ years). All of the participants completed a questionnaire that included demographic and clinical data, as well as information about any previous PFTs.
The participants then underwent spirometry in accordance with international recommendations. ${ }^{(3)}$

Of the 267 individuals studied, 167 (62.5\%) had never undergone PFTs. Therefore, for the majority of the participants, this project constituted the first evaluation of their pulmonary function. of the 167 participants who had never undergone PFTs, 84 were current smokers and 48 were former smokers. More importantly, 55.1\% presented with at least one respiratory symptom (such as dyspnea, expectoration, and wheezing). Despite presenting with these risk factors for a major respiratory disease, those participants had never sought medical attention. In addition, of the 167 participants who had never undergone PFTs, 32 $(19.2 \%)$ showed abnormal spirometric results and $110(65.6 \%)$ presented with respiratory symptoms, such as cough, dyspnea, and decreased exercise tolerance.

The underdiagnosis of COPD depends on various factors that might be affected by the current economic crisis, such as the problematic access to health care facilities due to financial problems, the lack of diagnostic facilities, and the absence of skilled, experienced staff. In addition, the low level of education associated with the economic downturn might result in a lack of information concerning risk factors and could even prevent a significant number of patients from identifying their symptoms.

Spirometry is an inexpensive, noninvasive test of ventilatory function and plays a critical role in the diagnosis of respiratory diseases. However, screening the general population would not be feasible. One group of authors suggested that patients with a high probability of having COPD be identified through the use of symptom-based questionnaires and subsequently screened with PFTs as needed. Delayed diagnoses and a large number of unnecessary spirometric tests would thus be avoided. ${ }^{(4)}$ 
During a financial crisis, when governments cannot invest in public health care and people can barely afford special examinations, consequently neglecting their health, spirometry should be accessible to all symptomatic patients in order to promote early diagnosis and prevent the progression of the disease, thereby reducing health costs. Screening tools, such as spirometry, should be made available to subjects at risk for respiratory diseases, such as elderly patients and smokers, with the aim of reducing the underdiagnosis of such diseases, which can be adequately treated when detected early.

\author{
Filio Kotrogianni \\ Resident in Respiratory Medicine, \\ Respiratory Medicine Department, \\ University Hospital of Larissa, \\ Larissa, Greece \\ Foteini Malli \\ Resident in Respiratory Medicine, \\ Respiratory Medicine Department, \\ University Hospital of Larissa, \\ Larissa, Greece
}

\section{Konstantinos 1 Gourgoulianis Professor of Respiratory Medicine, Faculty of Medicine, University of Thessaly, Larissa, Greece}

\section{References}

1. de Queiroz MC, Moreira MA, Rabahi MF. Underdiagnosis of COPD at primary health care clinics in the city of Aparecida de Goiânia, Brazil. J Bras Pneumol. 2012; 38(6):692-9.

2. World Health Association. The Financial Crisis and Global Health. Report of a High-Level Consultation, 19 January 2009. Geneva: World Health Organization; 2009.

3. Standardization of Spirometry, 1994 Update. American Thoracic Society. Am J Respir Crit Care Med. 1995;152(3): 1107-36.

4. Thorn J, Tilling B, Lisspers K, Jörgensen L, Stenling A, Stratelis G. Improved prediction of COPD in at-risk patients using lung function pre-screening in primary care: a real-life study and cost-effectiveness analysis. Prim Care Respir J. 2012;21(2):159-66. 